\title{
Dermatoscopia na Idade Pediátrica - Parte II: Dermatoses Infeciosas e Inflamatórias
}

\author{
Rita Pimenta' ', Sónia Fernandes', Lucero Noguera-Morel2, Paulo Filipe ${ }^{1,3,4}$, André Laureano Oliveira 1,3 \\ 'Centro Hospitalar Universitário Lisboa Norte EPE, Hospital de Santa Maria, Serviço de Dermatologia. Lisboa, Portugal \\ ${ }^{2}$ Hospital del Niño Jesús, Serviço de Dermatologia, Madrid, Espanha \\ 3Universidade de Lisboa, Faculdade de Medicina, Clínica Universitária de Dermatologia de Lisboa. Lisboa, Portugal \\ ${ }^{4}$ Instituto de Medicina Molecular. Lisboa, Portugal
}

RESUMO - A dermatoscopia é uma técnica não-invasiva, que permite aumentar a acuidade diagnóstica de diversas lesões cutâneas. Sendo um procedimento indolor, a dermatoscopia é particularmente útil nas crianças, poupando-as de biópsias e terapêuticas desnecessárias. Na parte Il deste artigo, destacamos a importância da dermatoscopia para diagnóstico e acompanhamento de dermatoses infeciosas e inflamatórias na idade pediátrica.

PALAVRAS-CHAVE - Criança; Dermatoscopia; Dermatopatias Infecciosas/diagnóstico; Doenças da Pele/diagnóstico; Inflamação.

\section{Dermoscopy in Pediatric Dermatology - Part II: Infectious and Inflammatory Skin Lesions}

ABSTRACT - Dermoscopy is a noninvasive technique that increases diagnostic accuracy of several skin lesions. Being painless, dermoscopy is particularly useful in children, sparing them from unnecessary biopsies and treatments. In part II of this article, we highlight the importance of dermoscopy for the diagnosis and follow-up of infectious and inflammatory skin disorders in pediatric patients. KEYWORDS - Child; Dermoscopy; Inflammation/diagnosis; Skin Diseases/diagnosis; Skin Diseases, Infectious/diagnosis.

\section{INTRODUÇÃO}

A dermatoscopia constitui uma ferramenta útil para o diagnóstico e seguimento de diversas lesões cutâneas. Trata-se de uma técnica não-invasiva, rápida e indolor, pelo que é facilmente aceite pelos doentes pediátricos, poupando-os a biópsias e tratamentos desnecessários. ${ }^{1}$ Neste artigo, destacamos a importância da dermatoscopia para avaliação de dermatoses infeciosas e inflamatórias na idade pediátrica.

\section{Dermatoses infeciosas}

\subsection{Escabiose}

A escabiose é uma infestação cutânea altamente contagiosa, provocada pelo ácaro Sarcoptes scabiei var. hominis, e cuja prevalência mundial está estimada em 300 milhões de casos. ${ }^{2}$ Geralmente, os doentes têm prurido intenso com agravamento noturno e as lesões primárias consistem em pequenas pápulas eritematosas e sulcos acarinos (Fig.s la,b).
Nas crianças pequenas, a cabeça, palmas e plantas, habitualmente poupadas nos adultos, estão geralmente afetadas. $^{2}$ Nesta faixa etária as lesões são mais inflamatórias, observando-se com maior frequência vesico-pústulas. ${ }^{3}$

O diagnóstico é fundamentalmente clínico, no entanto, a apresentação pode ser atípica e mimetizar outras dermatoses. ${ }^{1}$ Desta forma, a identificação microscópica dos ácaros, ovos ou fezes, após raspado cutâneo, pode confirmar o diagnóstico. ${ }^{4}$ Apesar do raspado cutâneo ser um procedimento simples, este pode causar ansiedade e desconforto nos doentes mais novos. ${ }^{2}$ Por outro lado, a dermatoscopia (Fig. 1c) permite, inclusivamente com baixa ampliação, a identificação do parasita (sinal da "asa-delta") e das suas galerias (padrão em "rasto de avião"), de forma não invasiva. O primeiro surge como uma estrutura triangular acastanhada, correspondendo à superfície anterior do ácaro (gnatossoma e patas anteriores), no final de um segmento linear
Correspondência: Rita Pimenta

Clínica Universitária de Dermatologia de Lisboa

Hospital de Santa Maria

Avenida Professor Egas Moniz

1649-028 Lisboa, Portugal

E-mail: ritapimenta@campus.ul.pt

DOI: https://dx.doi.org/10.29021/spdv.78.3.1209
Recebido/Received 2020/05/06

(C) Autor (es) (ou seu (s) empregador (es)) 2020 Revista SPDV. Reutilização permitida de acordo com CC BY-NC. Nenhuma reutilização comercial.

(c) Author(s) (or their employer(s)) 2020 SPDV Journal. Re-use permitted under CC BY-NC. No commercial re-use. 


\section{Educação Médica Contínua}

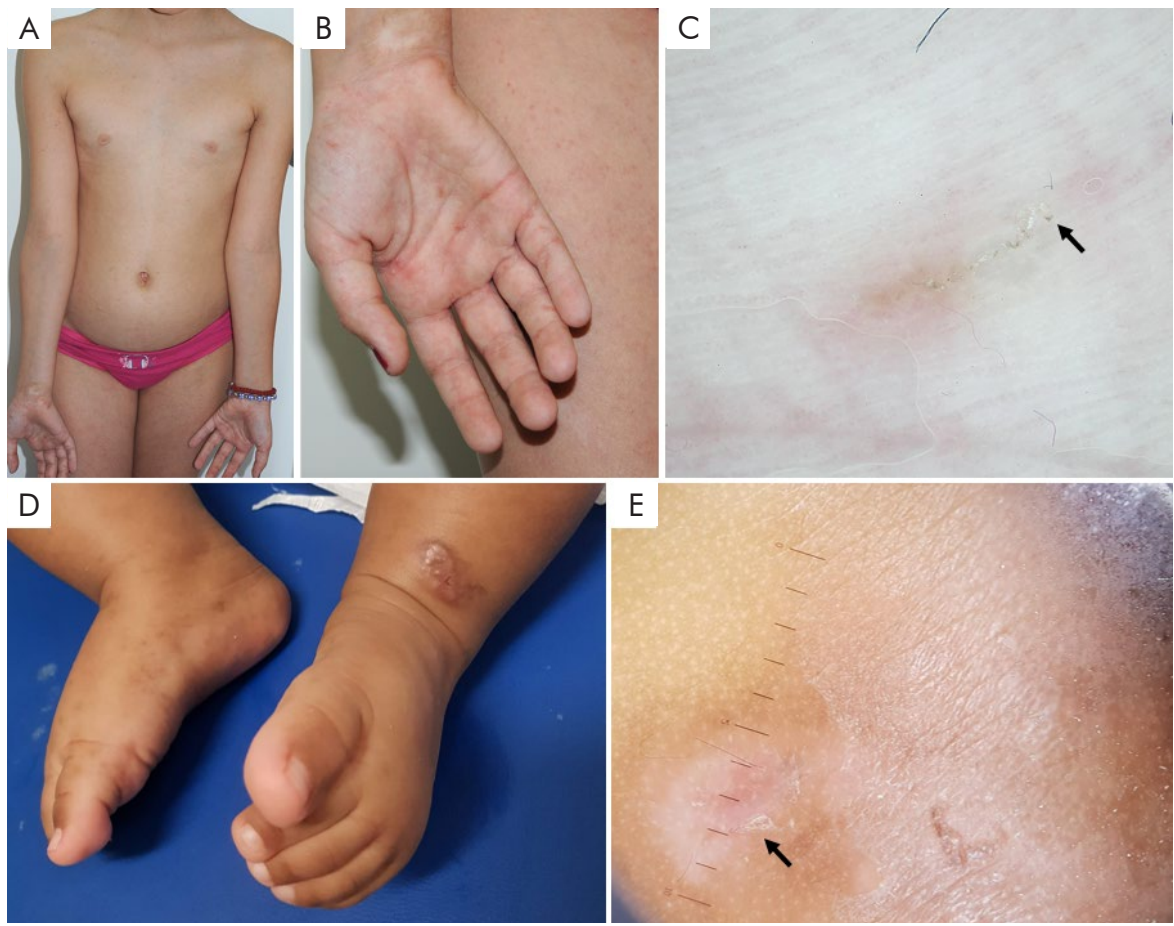

Figura 1 - Escabiose. (A) Criança de 9 anos com múltiplas pápulas eritematosas disseminadas pelo tronco e membros. (B) Pormenor das pápulas e sulcos acarinos na palma direita. (C) Dermatoscopia: segmento linear esbranquiçado, bem definido, com estrutura triangular acastanhada na extremidade (seta preta), correspondendo à galeria e ácaro, respetivamente. (D) Escabiose nodular em lactente de 6 meses. (E) Dermatoscopia: sinal "rasto de avião" (seta preta).

esbranquiçado, o sulco acarino.' Os mesmos aspetos foram descritos na escabiose nodular ${ }^{5,6}$ (Fig.s 1d,e), incluindo casos neonatais. Na sarna crostosa, foi recentemente descrito um novo sinal dermatoscópico ("noodle sign"), que representa sulcos acarinos agrupados. ${ }^{7}$

Estudos prospetivos verificaram que a dermatoscopia manual tem uma sensibilidade igual ou superior ( $83 \%$ - 91\%) ao exame microscópico convencional $(68 \%-90 \%)$, permitindo também aumentar a acurácia deste último. ${ }^{8}$ Contudo, tem uma menor especificidade ( $46 \%$ - $86 \%)$, em comparação com o raspado cutâneo (que é por definição 100\%), sendo, por vezes, difícil distinguir o sinal "rasto de avião" de artefactos, como escoriações. ${ }^{8}$

A videodermatoscopia, com maior capacidade de ampliação, proporciona ainda uma sensibilidade e especificidade superior, permitindo observar a estrutura dos ácaros com maior detalhe, os ovos, as fezes e, em alguns casos, a migração dos ácaros dentro das galerias. ${ }^{2,8}$ As fezes surgem como pequenos pontos castanhos ou esbranquiçados, enquanto os ovos apresentam-se como estruturas ovais translúcidas, semelhantes a um colar de pérolas. ${ }^{2,9}$

A dermatoscopia também é útil para avaliar a resposta ao tratamento. Cerca de duas semanas após a terapêutica escabicida, os ácaros começam a decompor-se e a sua aparência torna-se desfocada e indistinta à dermatoscopia. ' Além disso, a incapacidade de deteção do sinal da "asa-delta" em toda a superfície cutânea pode traduzir a eficácia do tratamento. ${ }^{4}$

\subsection{Pediculose do couro cabeludo}

A pediculose do couro cabeludo é uma infestação causada pelo ectoparasita Pediculus humanus capitis. ${ }^{10} \mathrm{~A}$ prevalência é variável, desde $1 \%$ a $20 \%$, surgindo preferencialmente nas crianças entre os 3 e 12 anos. ${ }^{10}$ Apesar do piolho adulto nem sempre ser identificado, pela sua cor translúcida e movimentos rápidos, o diagnóstico pode ser confirmado pela observação das lêndeas aderentes à haste capilar. ${ }^{1}$ Contudo, as escamas na dermite seborreica e noutras dermatoses descamativas do couro cabeludo, bem como resquícios de cosméticos, podem ser confundidos com as lêndeas. ${ }^{4}$ Com a dermatoscopia é possível diferenciá-los e monitorizar a resposta ao tratamento, distinguindo as lêndeas contendo ninfas viáveis das vazias. Na dermatoscopia, as lêndeas com ninfas (Fig. 2a) têm uma cor castanha e formato ovóide, enquanto as vazias (Fig. 2b) são translúcidas e têm uma extremidade livre aplanada, por vezes fissurada. ${ }^{4,10}$ Algumas contêm ninfas mortas, que surgem como uma área castanha focal num fundo translúcido.' Por outro lado, as pseudo-lêndeas destacam-se facilmente da haste capilar e na dermatoscopia caraterizam-se por estruturas esbranquiçadas e amorfas. ${ }^{4}$

\subsection{Larva migrans}

A larva migrans cutânea é uma dermatose parasitária, auto-limitada, que resulta da invasão e migração de larvas de nemátodes (Ancylostoma braziliense, Ancylostoma caninum, Uncinaria stenocephala, entre outros) na pele." $\mathrm{A}$ infestação 


\section{Educação Médica Contínua}
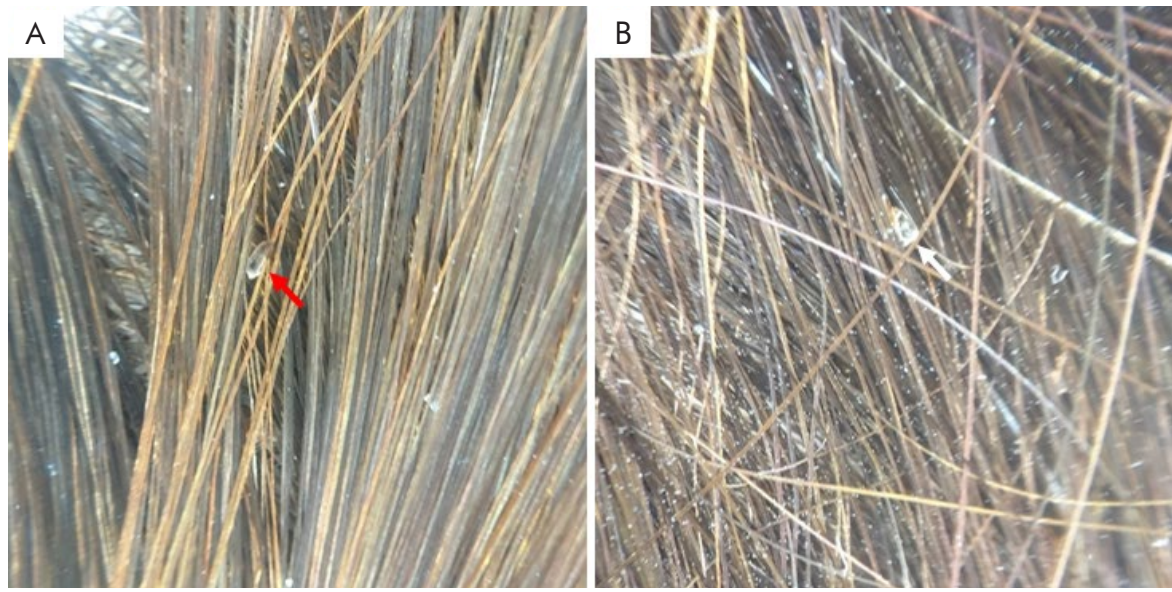

Figura 2 - Dermatoscopia de pediculose do couro cabeludo em adolescente de 16 anos. (A) Lêndea viável (seta vermelha), com formato ovóide e extremidade convexa, aderente à haste capilar. (B) Lêndea vazia (seta branca), translúcida e com extremidade livre aplanada.

é adquirida por contacto com solos contaminados com excrementos de animais infetados e é típica de países tropicais ou subtropicais. ${ }^{12}$ Clinicamente (Fig. 3a) carateriza-se por lesões pruriginosas, eritematosas, com trajeto serpiginoso ou linear, predominantemente localizadas nos pés. ${ }^{4,13}$ As larvas não atravessam a membrana basal, ficando confinadas à epiderme, onde migram a uma velocidade de 2 a $5 \mathrm{~cm}$ por dia. ${ }^{14}$ Apesar das manifestações caraterísticas, os exames auxiliares de diagnóstico podem ser necessários em casos atípicos. $\mathrm{Na}$ biópsia cutânea, os achados são inespecíficos e a larva é raramente visualizada. ${ }^{14} \mathrm{~A}$ dermatoscopia (Fig. 3b) permite a identificação de áreas homogéneas amarelo-acastanhadas, translúcidas, com distribuição serpiginosa, correspondendo à larva, enquanto que os "túneis epidérmicos" produzidos por estas, apresentam vasos em ponto. $4,12,13,15$

\subsection{Tinha do couro cabeludo}

A tinha do couro cabeludo é a infeção fúngica por dermatofitos mais comum em idade pediátrica, afetando fundamentalmente crianças pré-púberes. ${ }^{16} \mathrm{~A}$ apresentação clínica é variável, podendo manifestar-se como uma dermatose não-inflamatória, com áreas de alopécia (Fig. 4a,b) e/ou placas descamativas, ou como uma doença inflamatória, com placas ou nódulos dolorosos e supurativos. ${ }^{17}$

Embora o exame microscópico direto e a cultura micológica sejam os exames de referência para o diagnóstico da tinha do couro cabeludo, a tricoscopia (Fig. 4c,d) possibilita a sua identificação e tratamento imediato. ${ }^{1,13,18}$ Os achados dermatoscópicos mais frequentes e específicos consistem em cabelos em vírgula, saca-rolhas e código Morse. ${ }^{17-19}$ Os cabelos em vírgula constituem hastes pilosas curtas, de espessura uniforme e curvas, podendo adquirir a forma de saca-rolhas em doentes de raça negra ou com cabelo encaracolado. ${ }^{17,19}$ Estão associados tanto ao parasitismo endotrix, como ectotrix. ${ }^{1} 18$ Nos cabelos em código Morse observam-se bandas transversais alternadas, esbranquiçadas e acastanhadas. ${ }^{17}$
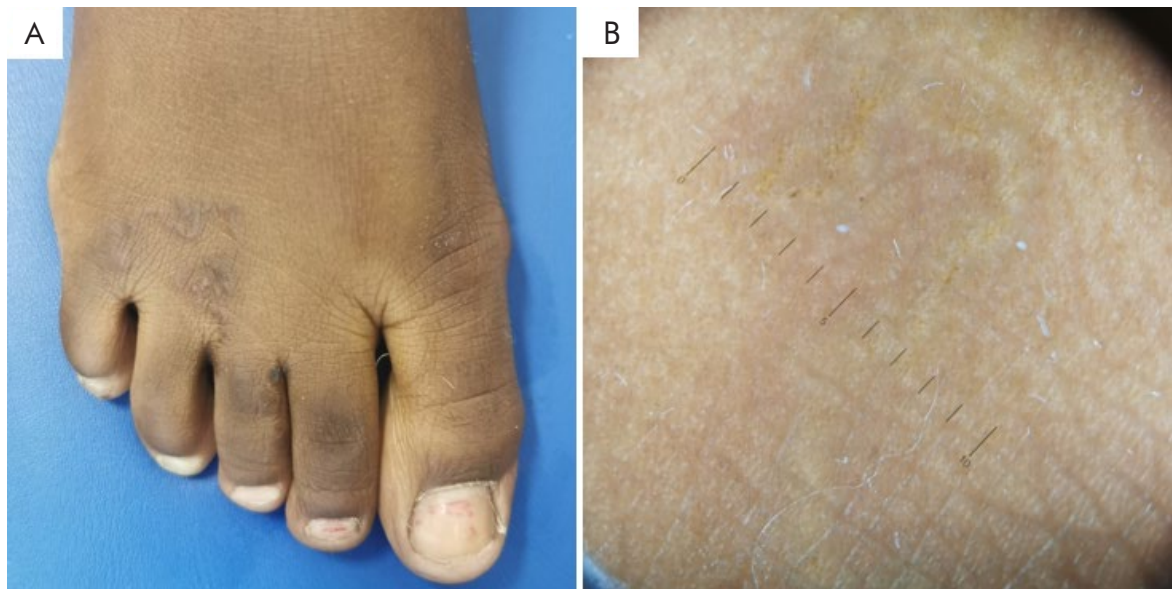

Figura 3 - Larva migrans. (A) Lesão serpiginosa no dorso do pé direito de criança com 8 anos. (B) Dermatoscopia: larva migrans com aspeto serpiginoso e segmentado. 


\section{Educação Médica Contínua}
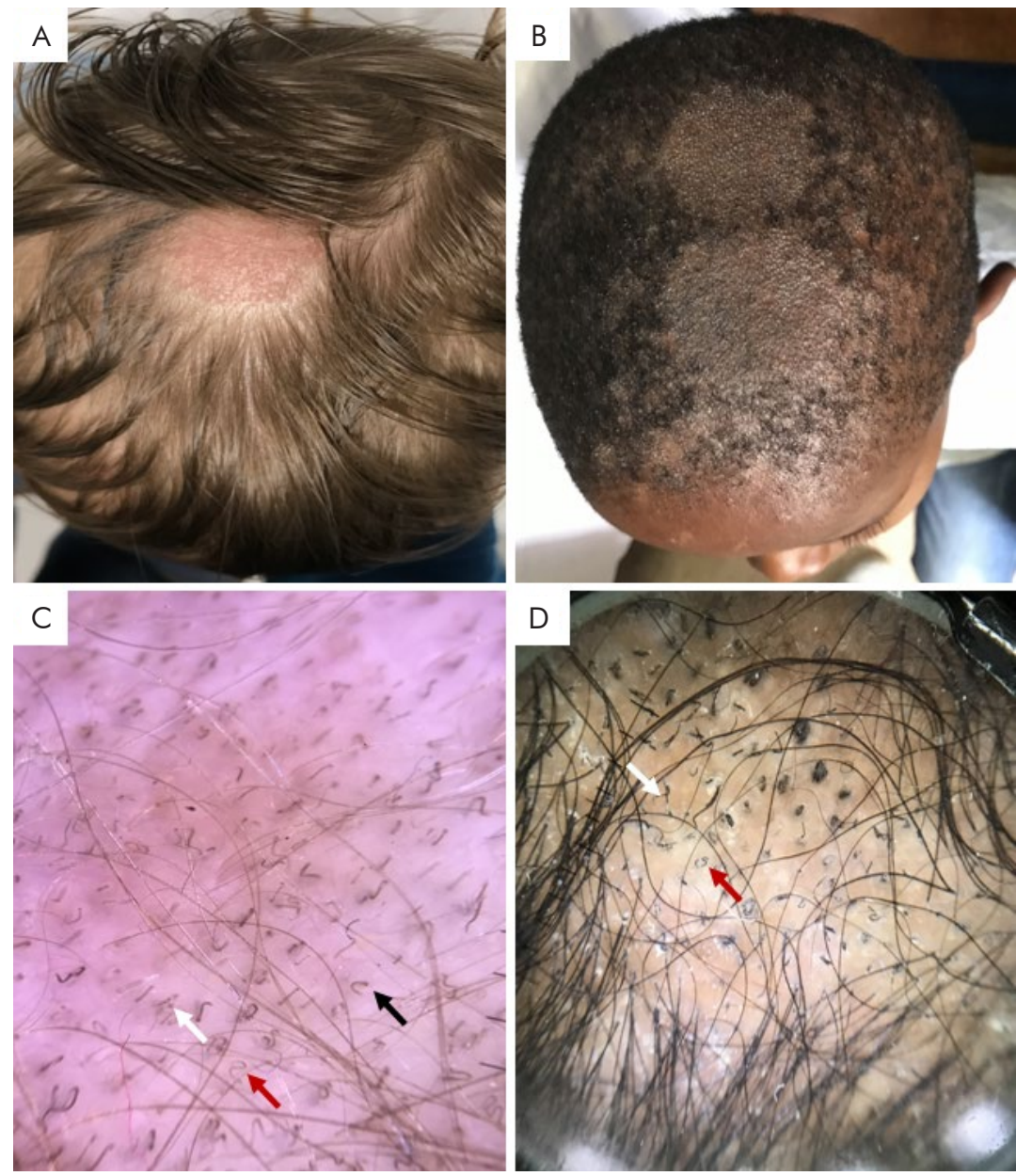

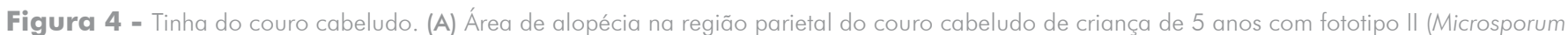

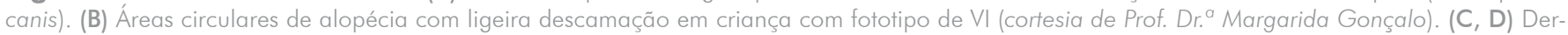
matoscopia: cabelos em vírgula (seta preta), saca-rolhas (seta vermelha) e código Morse (seta branca).

Em grande ampliação (x100), as bandas mais claras surgem como espaços vazios, representando áreas de invasão fúngica, onde a haste é menos resistente e facilmente quebrável. ${ }^{17}$ Podem também ser observados cabelos em zigzag, caraterizados por hastes com sucessivas dobras em ângulo agudo. ${ }^{18}$ Verificou-se ainda que a associação entre descamação perifolicular com cabelo distrófico ou fragmentado pode representar um padrão específico da tinha do couro cabeludo. ${ }^{20}$ Outros achados inespecíficos incluem cabelos quebrados, pontos pretos e cabelos em tufos. ${ }^{21}$

\subsection{Verrugas virais}

As verrugas virais são proliferações epiteliais benignas da pele e das mucosas, resultantes da infeção pelo vírus do papiloma humano (HPV). ${ }^{22}$ As verrugas cutâneas são frequentes em idade escolar, com uma prevalência estimada entre $5 \%$ e $21,7 \%$, enquanto as anogenitais são menos comuns, desconhecendo-se a prevalência exata na idade pediátrica. ${ }^{22}$ Nestas últimas, o abuso sexual deve ser excluído, contudo, as principais vias de transmissão nesta faixa etária são a perinatal e a auto ou hetero-inoculação. ${ }^{23,24}$ De acordo com a sua localização anatómica e morfologia, as verrugas são classificadas em vulgares, plantares, planas e genitais ou condilomas.

As verrugas têm uma aparência clínica distinta e são habitualmente diagnosticadas a olho nu. No entanto, a sua identificação pode ser difícil nos estadios iniciais e após tratamento. ${ }^{25} \mathrm{~A}$ dermatoscopia facilita o diagnóstico e os achados variam com o subtipo.,13 As verrugas vulgares (Fig. 5) exibem tipicamente múltiplas papilas centradas por um vaso (em ponto ou em gancho) circundado por um halo esbranquiçado (padrão "frogspawn"). ${ }^{13,25}$ Os vasos trombosados surgem como glóbulos pretos e, por vezes, estão presentes pequenos pontos hemorrágicos. ${ }^{1,13}$

As verrugas plantares revelam, à dermatoscopia, áreas verrucosas amareladas, com múltiplos pontos e/ou estrias lineares, vermelhas ou pretas, irregularmente distribuídas, correspondendo a microhemorragias, potenciadas pela pressão exercida sobre a região plantar. $4,13,22$ Estes aspetos permitem distinguir as verrugas das calosidades plantares, que não 


\section{Educação Médica Contínua}
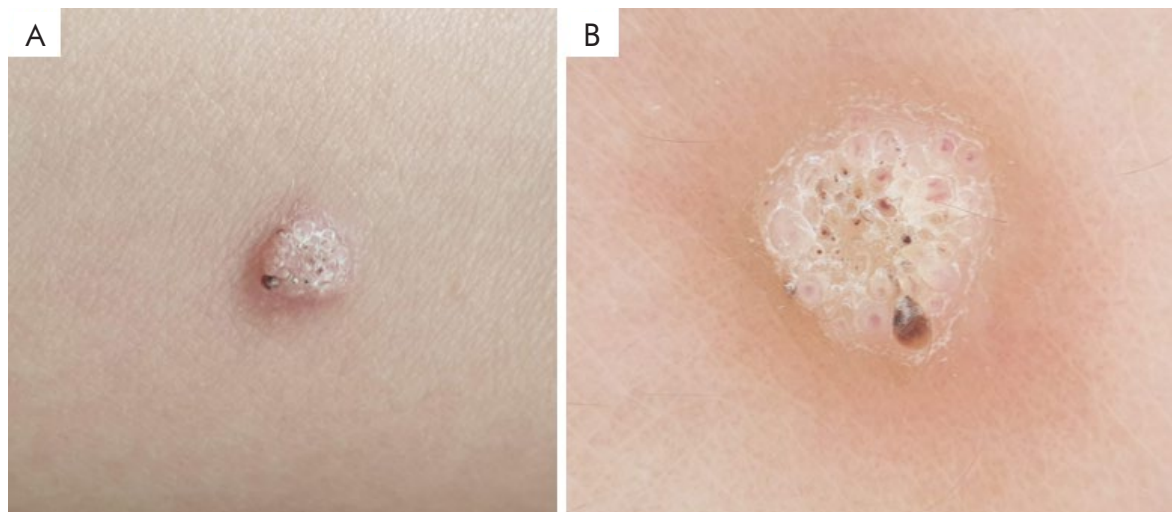

Figura 5 - Verruga vulgar. (A) Pápula hiperqueratótica na perna esquerda de criança de 7 anos. (B) Dermatoscopia: papilas agrupadas com vasos ou pontos hemorrágicos, circundados por halo esbranquiçado.

apresentam microhemorragias, mas exibem uma área homogénea central, avermelhada ou azulada. ${ }^{1}$ As verrugas plantares devem ainda ser diferenciadas do melanoma acral. ${ }^{13}$

A dermatoscopia das verrugas planas demonstra pequenos pontos vermelhos regularmente distribuídos num fundo castanho claro ou amarelo. ${ }^{22}$ Estes achados possibilitam a diferenciação com comedões ou foliculites, caraterizados por uma estrutura redonda central, branca ou amarela, que corresponde a um rolhão de queratina ou pus no infundíbulo. ${ }^{22}$

Nos condilomas acuminados, a dermatoscopia pode ser útil para distingui-los de patologias não infeciosas, como as pápulas peroladas do pénis, papilomatose vestibular e grânulos de Fordyce. ${ }^{22} \mathrm{O}$ padrão em mosaico é o mais comum e consiste numa rede esbranquiçada que circunscreve áreas centradas por vasos em ponto ou glomerulares dilatados. ${ }^{26}$ Por vezes, os condilomas revelam múltiplas projeções esbranquiçadas e irregulares, contendo vasos alongados e dilatados, geralmente mais pronunciados à periferia. ${ }^{22,26}$

A dermatoscopia pode também avaliar a eficácia do tratamento das verrugas vulgares e plantares. $O$ desaparecimento das microhemorragias e do padrão vascular pressupõe um baixo risco de recorrência. ${ }^{1}$

\subsection{Molusco contagioso}

O molusco contagioso ( $M C$ ) é uma infeção cutânea viral causada por um poxvirus. ${ }^{27} \mathrm{~A}$ sua incidência anual é de $2 \%$ a $20 \%$, sendo muito comum na infância, principalmente entre os 2 e os 5 anos de idade. ${ }^{27,28}$ Clinicamente (Fig.s 6a,b), manifesta-se por pequenas pápulas umbilicadas, de cor rosada ou branco pérola, únicas ou múltiplas. ${ }^{27}$ Embora a maioria das lesões de MC seja facilmente diagnosticada com base na sua apresentação clínica, algumas são indistinguíveis de quistos de milia, verrugas virais ou até de nevos de Spitz. ${ }^{1}$ A dermatoscopia pode ser útil ao demonstrar as caraterísticas típicas desta infeção. No molusco contagioso observa-se uma estrutura central redonda ou polilobulada, branca ou amarelada, circundada por uma coroa de vasos lineares e finos, por vezes ramificados, mas que não intersetam o centro ${ }^{27,29,30}$ (Fig.s 6c,d). A estrutura central corresponde histologicamente à hiperplasia endofítica e lobular da epiderme, enquanto o padrão vascular pode ser explicado pela compressão e separação dos vasos pelos septos fibrosos. ${ }^{1}$ Por outro lado, as lesões irritadas ou traumatizadas estão frequentemente associadas a vasos punctiformes. ${ }^{29}$ Estão ainda descritas estruturas brancas com disposição circular, semelhantes a um trevo de quatro folhas, que podem ser observadas à luz polarizada. ${ }^{13,30}$

\section{Dermatoses inflamatórias}

\subsection{Psoríase}

A psoríase é uma doença inflamatória crónica e imunologicamente mediada, que atinge cerca de $0,7 \%$ a $2 \%$ das crianças. ${ }^{31}$ Nesta faixa etária, a apresentação mais frequente é a psoríase em placas e o couro cabeludo é a principal área afetada. ${ }^{32}$ As lesões tendem a ser de menor espessura e dimensão em comparação com os adultos. ${ }^{31}$ Outras variantes comumente observadas na idade pediátrica são a psoríase gutata (Fig.s 7a,b) e inversa. ${ }^{31,32}$

O diagnóstico de psoríase nas crianças pode representar um desafio, especialmente nas formas localizadas (psoríase palmoplantar, genital, ou do couro cabeludo). ${ }^{19}$ Os principais diagnósticos diferenciais incluem a dermite atópica, a pitiríase rubra pilar, a pitiríase rosada, as dermatofitias e a dermite seborreica. ${ }^{31} \mathrm{O}$ exame histopatológico, embora específico, raramente é realizado nas crianças devido ao seu caráter invasivo $^{32}$ Por outro lado, vários estudos demonstraram a utilidade da dermatoscopia no diagnóstico da psoríase através da identificação de alterações específicas na microvasculatura cutânea, que se correlacionam com alterações histopatológicas precoces. ${ }^{19}$

A dermatoscopia com baixa ampliação (10x) mostra a presença de vasos em ponto ou glóbulos regularmente distribuídos num fundo vermelho claro. ${ }^{19,33} \mathrm{Em}$ maior ampliação ( $\geq 100 x)$, os pontos/glóbulos vermelhos surgem como capilares dilatados e torcidos, descritos como "bushy" ou "basket-like". ${ }^{33}$ Cada capilar mede cerca de 50 a $110 \mu \mathrm{m}$ de diâmetro, sendo significativamente superior ao observado na pele sem lesões $(15-25 \mu \mathrm{m}){ }^{31}$ Os capilares "bushy-like" 


\section{Educação Médica Contínua}
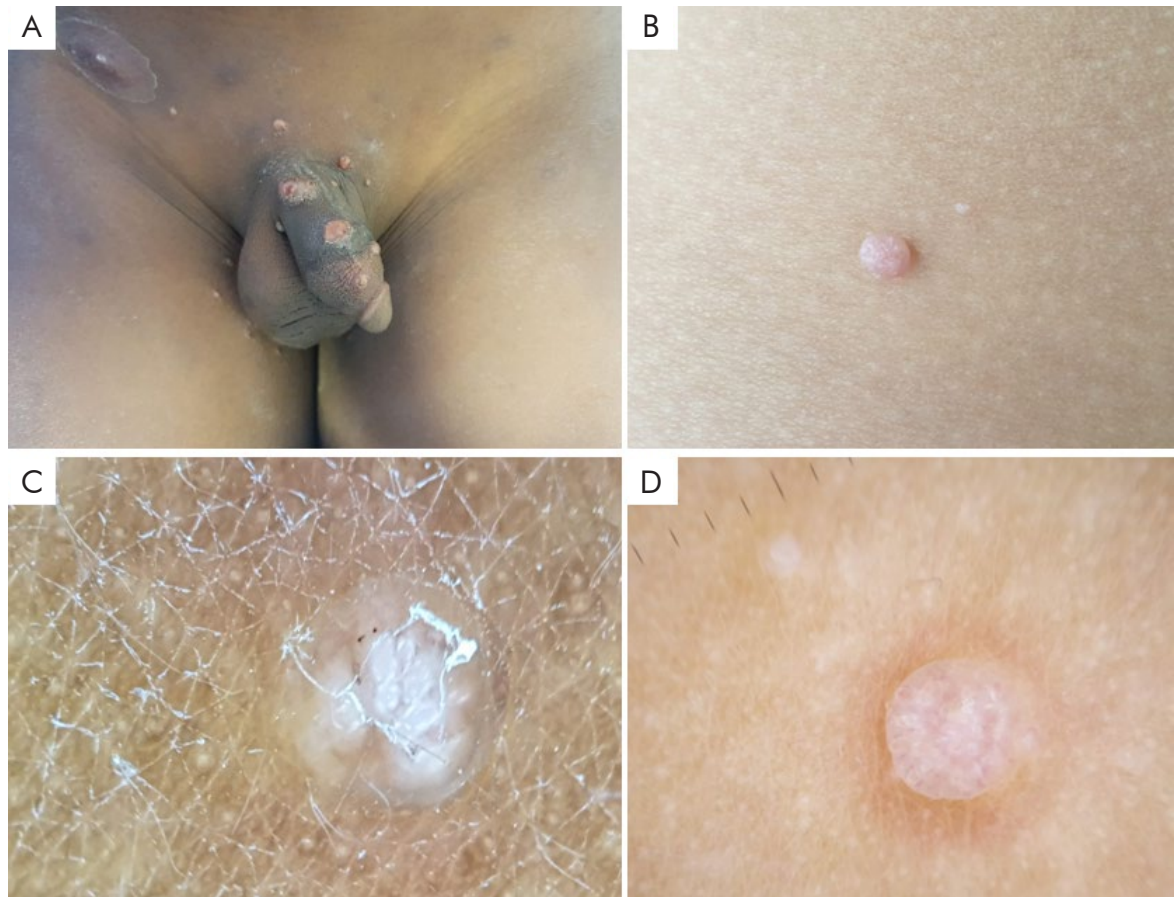

Figura 6 - Molusco contagioso. (A) Múltiplas pápulas branco-rosadas com umbilicação central na região genital de criança de 8 anos, após contágio por auto-inoculação. (B) Pápula rosada umbilicada no braço direito de criança de 4 anos. Padrões dermatoscópicos do molusco contagioso: (C) Estrutura branca central polilobulada, com estruturas semelhantes a trevo de quatro folhas. (D) Estrutura branca polilobulada, circundada por vasos lineares e ramificados.

predominam no centro da lesão, enquanto na periferia observam-se ansas capilares alongadas, em forma de gancho, paralelas à superfície cutânea. ${ }^{33}$ Este padrão, quando distribuído uniformemente por toda a lesão, é caraterístico da psoríase e corresponde histologicamente aos capilares dilatados nas papilas dérmicas alongadas. ${ }^{31}$

O mesmo padrão vascular pode ser observado nas lesões localizadas no couro cabeludo, na região genital e nas palmas e plantas. ${ }^{33} \mathrm{Na}$ psoríase palmoplantar, os capilares estão dispostos linearmente ao longo dos sulcos. ${ }^{33}$ De salientar que a hiperqueratose pode dificultar a visualização das alterações vasculares. ${ }^{31}$
Os pontos vermelhos e os capilares "bushy-like" podem ainda ser encontrados noutras dermatoses inflamatórias (p.e. eczema, pitiríase rósea, pitiríase rubra pilar), no entanto, de forma, muitas vezes, isolada e sem distribuição homogénea. ${ }^{32}$ Num estudo realizado por Lallas et al, verificou-se que a combinação de escamas brancas difusas com vasos em ponto com distribuição regular e sobre um fundo vermelho claro, permitiu o diagnóstico de psoríase em placas com uma sensibilidade de $84,9 \%$ e uma especificidade de $88,0 \% .^{33}$

Na psoríase ungueal, a dermatoscopia permite analisar com maior pormenor alterações da matriz (picotado) e do leito (onicólise, manchas salmão e hemorragias em estilhaço),
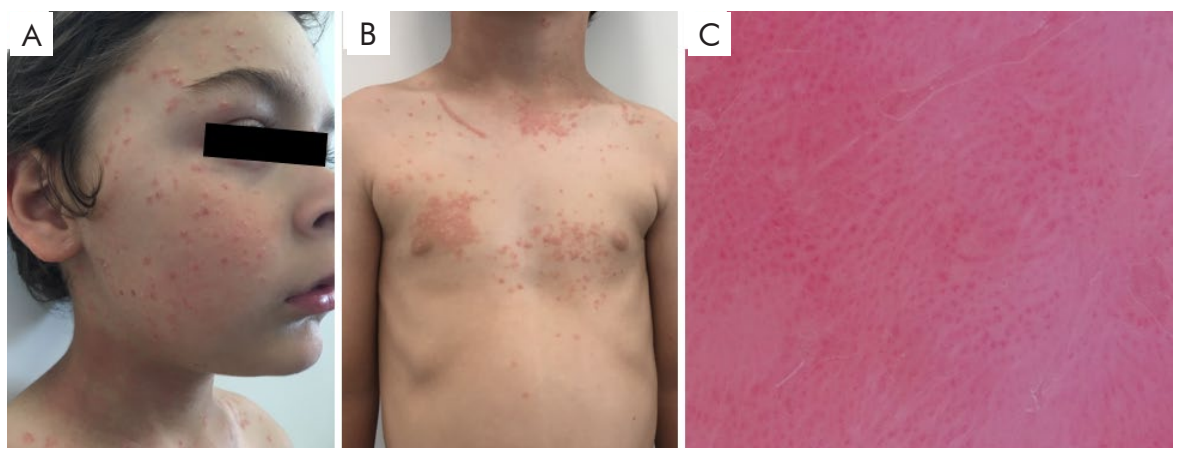

Figura 7 - Psoríase gutata. Criança de 5 anos com múltiplas pápulas e placas eritemato-descamativas na face (A), tronco (B) e membros, após faringite. Notar fenómeno de Koebner na região infra-clavicular direita. (C) Dermatoscopia: vasos em ponto regularmente distribuídos num fundo vermelho claro. 


\section{Educação Médica Contínua}

assim como detetar alterações vasculares do hiponíquio e da prega ungueal proximal. ${ }^{34,35} \mathrm{Na}$ onicólise, a onicoscopia mostra um bordo vermelho-alaranjado em redor do descolamento e as manchas salmão têm forma e tamanho irregular, com cor vermelha ou alaranjada. ${ }^{35}$

A dermatoscopia tem sido também utilizada para monitorização terapêutica. 1,33 $\mathrm{O}$ diâmetro dos capilares "bushy-like" diminui com os tratamentos tópicos e sistémicos, e o aparecimento de pontos purpúricos foi descrito como um sinal de boa resposta aos agentes biológicos. ${ }^{33}$ Por outro lado, o uso excessivo de corticosteróides tópicos pode ser identificado pela observação de linhas vermelhas em redor e sobre as placas psoriáticas, tratando-se de um sinal de atrofia cutânea. ${ }^{33}$

\subsection{Líquen plano}

O líquen plano (LP) é uma dermatose inflamatória idiopática que além da pele, pode afetar as mucosas, folículos pilosos e unhas. ${ }^{36}$ Surge geralmente na idade adulta, mas $0,1 \%$ a $10 \%$ dos casos correspondem a crianças. ${ }^{36}$ Nesta faixa etária, a apresentação clássica é a mais frequente, mas estão também descritas numerosas variantes, incluindo - LP agudo, hipertrófico, linear, anular, pigmentoso, actínico (Fig.s 8a,b), atrófico, erosivo, penfigóide e bolhoso. ${ }^{37-39} \mathrm{O}$ envolvimento da mucosa oral (4\% - 39\%), couro cabeludo $(2 \%-11,8 \%)$ e unhas $(1,7 \%-33,3 \%)$ é incomum. ${ }^{37}$

Nos casos com apresentação atípica, o exame histopatológico pode ser necessário para confirmar o diagnóstico. Porém, a dermatoscopia possibilita a identificação de estruturas que podem ser inaparentes a olho nu. As estrias de Wickham, patognomónicas, surgem como linhas branco-pérola, branco-azulado (especialmente em fototipos altos) ou amareladas, com várias morfologias, nomeadamente reticular (a mais comum), linear, anular, radial, foliácea (ramificações secundárias de uma estria central), e "em céu estrelado" (pontos brancos foliculares agrupados). ${ }^{36,40}$ As estrias correspondem à ortoqueratose compacta sobre a hipergranulose em " $\mathrm{v}$ ", estando normalmente presentes nas lesões ativas. ${ }^{1,36}$
Outros achados dermatoscópicos são: o fundo violáceo ou avermelhado; vasos em ponto ou lineares (correspondendo à dilatação dos vasos papilares e subpapilares, respetivamente), sobretudo na periferia das estrias, ou com padrão perifolicular ou difuso; pontos brancos/amarelos; e estruturas pigmentadas (pontos, glóbulos e áreas reticulares ou homogéneas). ${ }^{36}$

O padrão dermatoscópico varia com a evolução da doença. Nas lesões iniciais, observam-se estrias de Wickham subtis sobre um fundo avermelhado. ${ }^{36}$ Posteriormente, as estrias e os vasos tornam-se proeminentes, tendendo a desaparecer nas lesões em regressão. ${ }^{36}$ Nesta fase, predominam as estruturas pigmentadas com um padrão homogéneo ou granular. $^{1,36} \bigcirc$ padrão homogéneo castanho corresponde à incontinência pigmentar na derme e é geralmente transitório. 1,36 Por outro lado, o padrão granular, representado por múltiplos pontos e grânulos cinzentos ou acastanhados, correlaciona-se com a presença de melanófagos na derme e tende a ser mais persistente. ${ }^{1,36}$

Alguns subtipos do LP apresentam variações dermatoscópicas. O LP hipertrófico exibe pseudo-comedões preenchidos por rolhões de queratina amarela ("corn pearls"), que correspondem aos infundíbulos dilatados com ortoqueratose. ${ }^{36}$ No LP pigmentoso e actínico (Fig. 8c), as estrias e os vasos são menos frequentes, predominando as estruturas pigmentadas. ${ }^{36,41}$ No LP agudo, as estrias também são incomuns, mas os vasos são proeminentes. ${ }^{41} \mathrm{O}$ líquen plano pilar, raro na infância, demonstra descamação branca e eritema perifolicular, vasos arboriformes e áreas vermelho-leitoso cobertas por pontos brancos irregulares. ${ }^{37,38,40}$ Quando há envolvimento das unhas, a onicoscopia facilita a visualização das fissuras longitudinais da lâmina ungueal. ${ }^{35}$

\subsection{Dermatoses purpúricas pigmentadas}

O líquen aureus (LA) e a púrpura anular telangiectásica de Majocchi (PATM) são dois subtipos incomuns de dermatoses purpúricas pigmentadas, que afetam predominantemente crianças, adolescentes ou adultos jovens. ${ }^{42}$
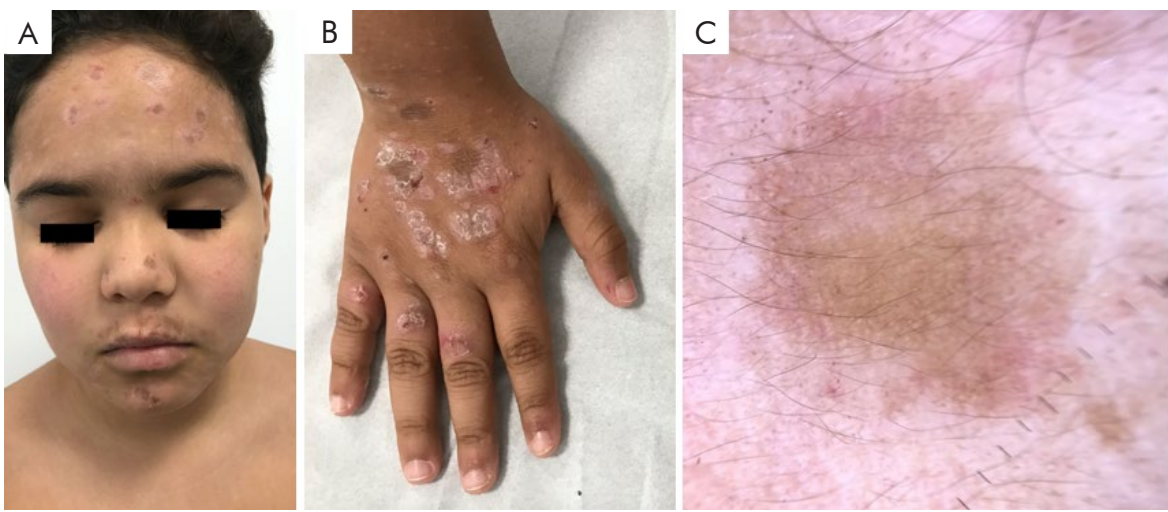

Figura 8 - Líquen plano actínico (forma anular). Criança de 9 anos com múltiplas placas anulares com centro acastanhado atrófico e bordo eritemato-descamativo na face (A) antebraços e dorso das mãos (B). (C) Dermatoscopia: padrão homogéneo acastanhado, com escassas estrias de Wickham e grânulos castanhos na periferia. 


\section{Educação Médica Contínua}

Clinicamente, o LA manifesta-se por máculas ou pápulas liquenóides, de coloração castanho-dourada a castanho-arroxeada, que coalescem em placas bem delimitadas, frequentemente solitárias e preferencialmente localizadas nas pernas. ${ }^{42,43}$ A PATM carateriza-se por um número variável de placas eritematosas anulares, com petéquias e telangiectasias finas e curtas nos bordos, habitualmente simétricas e localizadas nas extremidades proximais dos membros inferiores. ${ }^{42}$ Ambas as dermatoses são assintomáticas ou levemente pruriginosas, e geralmente têm uma evolução crónica. ${ }^{42,43}$

O diagnóstico é clínico, podendo ser necessário exame histopatológico em caso de dúvida diagnóstica. Nos últimos anos, a dermatoscopia tem também auxiliado no seu diagnóstico diferencial. As dermatoses purpúricas pigmentadas apresentam achados dermatoscópicos semelhantes (Fig.s 9d,e), consistindo em pontos, glóbulos ou manchas, eritemato-purpúricos, não branqueáveis, arredondados ou irregulares, sobre um fundo vermelho-acastanhado ou vermelho-acobreado, correspondendo histologicamente à extravasão de eritrócitos e deposição de hemossiderina na derme papilar, respetivamente. ${ }^{19,42}$ Este último aspeto dermatoscópico tende a ser difuso e proeminente no líquen aureus, e focal e subtil na PATM. ${ }^{2}$ Adicionalmente, na PATM podem observar-se telangiectasias, em virtude de um aumento pronunciado de vasos dilatados. ${ }^{42}$ Por outro lado,
- LA pode mostrar pontos cinzentos e uma rede castanho-acinzentada, correspondendo a macrófagos repletos de hemossiderina na derme e a hiperpigmentação da camada basal com incontinência pigmentar na derme superior, respetivamente. ${ }^{42,43}$

\subsection{Alopécia areata}

A alopécia areata é uma forma de alopécia não cicatricial, de etiologia autoimune, cuja evolução é imprevisível. ${ }^{44}$ Trata-se de uma patologia comum em idade pediátrica, com uma prevalência estimada de 0,63\% nesta faixa etária, e que representa cerca de $20 \%$ de todos os casos de alopécia. ${ }^{45,46}$ Carateriza-se tipicamente por áreas de alopécia ovaladas, bem circunscritas, com atingimento preferencial do couro cabeludo (Fig. 10a), mas que podem surgir em qualquer área pilosa. ${ }^{44}$

O diagnóstico baseia-se na observação clínica, no entanto, outras causas de alopécia podem apresentar manifestações semelhantes, como a tinha do couro cabeludo e tricotilomania. ${ }^{44}$ A tricoscopia (Fig. 10b) pode ser útil quer para diagnóstico, quer para avaliação da atividade da doença. ${ }^{47}$ Os achados tricoscópicos mais comuns da alopécia areata nas crianças são os óstios foliculares vazios e os cabelos velus, observados em cerca de $80 \%$ e $70 \%$ dos casos, respetivamente. ${ }^{45}$

Os óstios amarelos na alopécia areata são abundantes
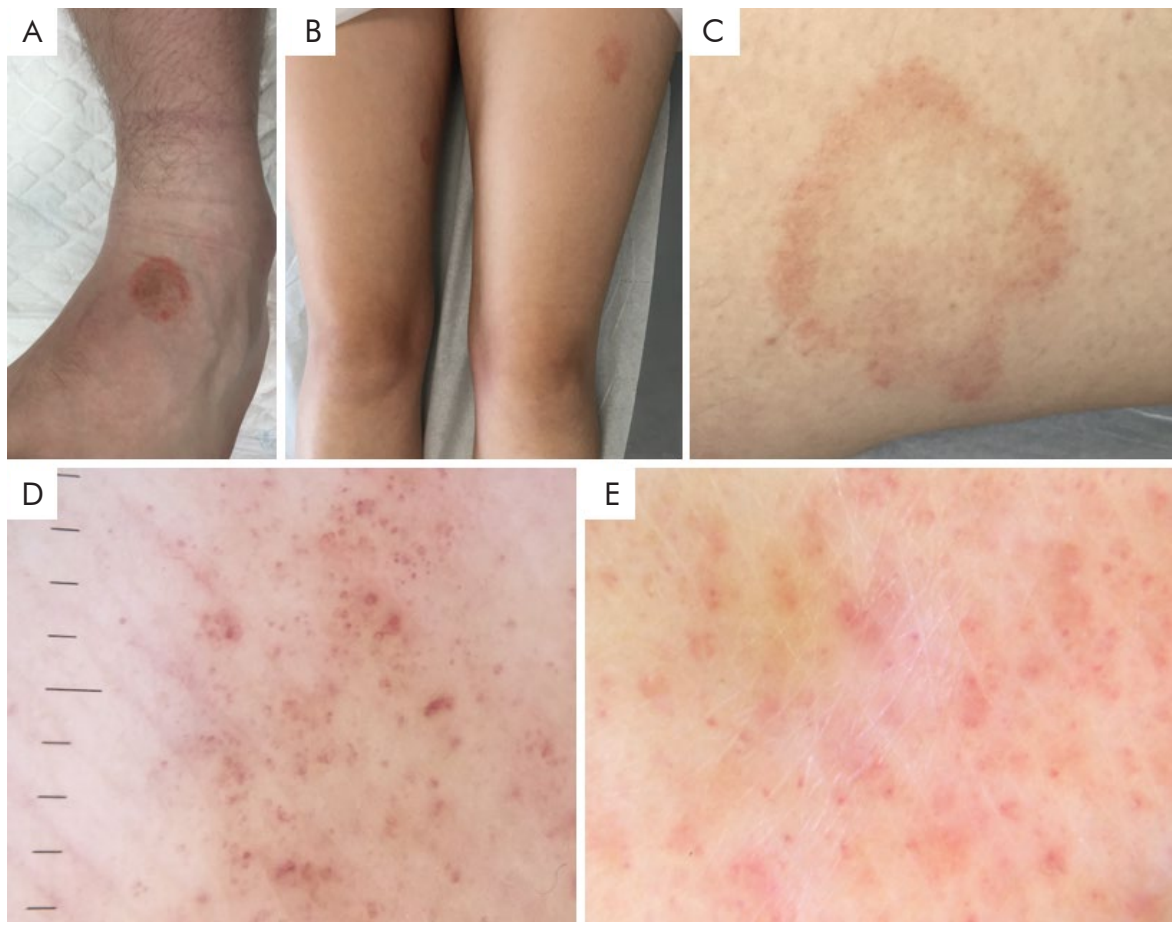

Figura 9 - Dermatoses purpúricas pigmentadas. (A) Placa acobreada (líquen aureus) no dorso do pé esquerdo de adolescente de 17 anos. (B) Placa eritematosa (púrpura anular telangiectásica de Majocchi) na face externa da coxa esquerda de doente de 16 anos. (C) Pormenor da lesão anterior, com configuração anular. Dermatoscopia do líquen aureus (D) e da púrpura anular telangiectásica de Majocchi (E): pontos e glóbulos purpúricos num fundo vermelho-acastanhado, mais proeminente e difuso no líquen aureus. 


\section{Educação Médica Contínua}
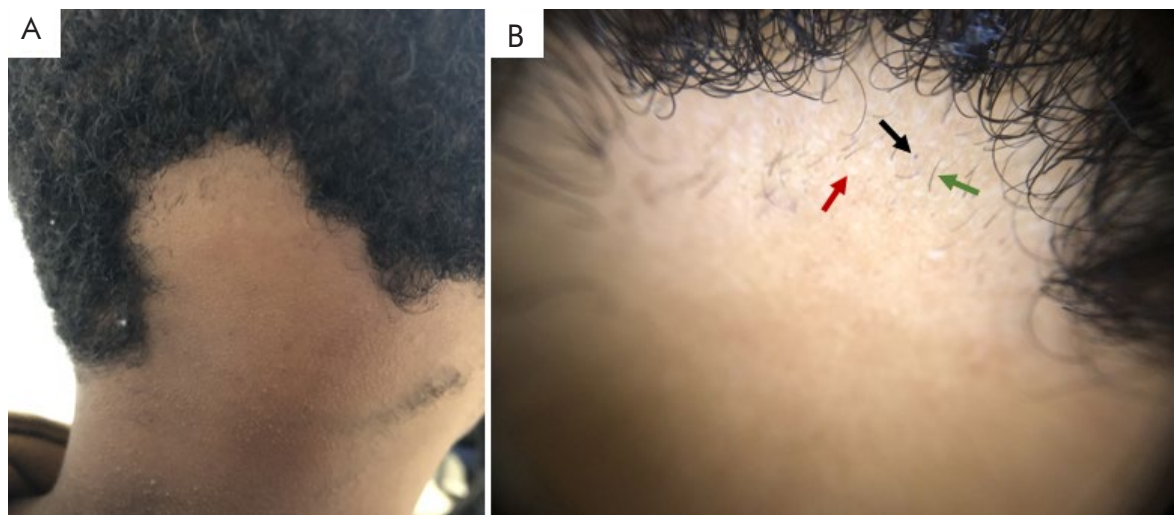

Figura 10 - Alopécia areata. (A) Área de alopécia, bem circunscrita, na região occipital em criança de 10 anos. $\bigcirc$ exame micológico excluiu tinha do couro cabeludo. (B) Dermatoscopia: óstios foliculares vazios (seta vermelha), óstios pretos (seta preta) e cabelos em ponto de exclamação (seta verde).

e têm geralmente uma distribuição regular. ${ }^{44,45}$ Contudo, são menos frequentes nas crianças em comparação com os adultos, sendo descritos em $40 \%$ a $100 \%$ dos doentes. ${ }^{45,47}$ Estes correspondem a orifícios foliculares dilatados preenchidos por rolhões de sebo e queratina, pelo que são raros nas crianças pequenas devido à baixa atividade das glândulas sebáceas. ${ }^{45,47}$ Além disso, os óstios amarelos nas crianças são predominantemente amarelo-claros, ao passo que nos adultos são tipicamente amarelo-acastanhados. ${ }^{45}$

Outros achados observados são os óstios foliculares pretos, os cabelos quebrados, em ponto de exclamação, em cone e triangulares, e as constrições Pohl-Pinkus, que predominam na doença ativa. ${ }^{44,47}$ Os óstios pretos correspondem a cabelos fraturados ao nível do couro cabeludo, e diferem dos visualizados na tinha do couro cabeludo e tricotilomania pela uniformidade no diâmetro e forma. ${ }^{47} \mathrm{Na}$ tricotilomania observam-se também cabelos quebrados, mas com diferentes comprimentos, ao contrário da alopécia areata. ${ }^{18}$

Os cabelos em ponto de exclamação e em cone caraterizam-se por uma porção distal mais espessa e escura que a proximal. ${ }^{47}$ Os primeiros têm cerca de 1 a $5 \mathrm{~mm}$ comprimento, enquanto os últimos são mais longos, não se identificando a porção distal na dermatoscopia. ${ }^{47}$

Os cabelos triangulares, recentemente descritos, têm uma terminação pontiaguda e uma haste proximal oculta sob um véu branco-acinzentado. ${ }^{45}$ As constrições Pohl-Pinkus correspondem a áreas da haste capilar com espessura diminuída, que resultam da supressão repetida e de forma rápida da atividade metabólica e mitótica do folículo. ${ }^{47}$

A tricoscopia viabiliza ainda monitorizar a evolução da doença e sua resposta ao tratamento. Os cabelos velus e sua transformação em cabelos terminais, os cabelos em recrescimento e o sinal peripilar branco predominam na fase de remissão. ${ }^{47}$ Os cabelos em recrescimento (em círculo ou em crescimento vertical) são mais comuns nas crianças em comparação com os adultos, o que pode resultar de maior crescimento capilar, assim como de remissões espontâneas mais frequentes neste grupo. ${ }^{45,47}$

\section{CONCLUSÃO}

Os procedimentos de diagnóstico invasivos e/ou semi-invasivos são muitas vezes difíceis de realizar na população pediátrica. Desta forma, a dermatoscopia é uma técnica de imagem útil, fácil e rápida de utilizar na prática clínica, auxiliando o diagnóstico e permitindo o acompanhamento de uma variedade de dermatoses.

Conflitos de interesse: Os autores declaram não possuir conflitos de interesse.

Suporte financeiro: $O$ presente trabalho não foi suportado por nenhum subsídio ou bolsa.

Proveniência e revisão por pares: Não comissionado; revisão externa por pares.

Conflicts of interest: The authors have no conflicts of interest to declare.

Financing support: This work has not received any contribution, grant or scholarship.

Provenance and peer review: Not commissioned; externally peer reviewed.

\section{ORCID}

Rita Pimenta

https://orcid.org/0000-0003-4649-7350

Sónia Fernandes

https://orcid.org/0000-0002-6936-2048

Lucero Noguera-Morel

https://orcid.org/0000-0001-5694-8536

Paulo Filipe

https://orcid.org/0000-0001-6917-527X

André Laureano Oliveira

https://orcid.org/0000-0002-9545-3736

\section{REFERÊNCIAS}

1. Haliasos EC, Kerner M, Jaimes N, Rudnicka L, Zalaudek I, Malvehy J, et al. Dermoscopy for the Pediatric 
Dermatologist Part I: Dermoscopy of Pediatric Infectious and Inflammatory Skin Lesions and Hair Disorders. Pediatr Dermatol. 2013; 30:163-71. doi: 10.1111/ pde. 12097.

2. Micali G, Giuffrida G, Quattrocchi E, Lacarrubba F. Scabies. In: Micali G, Lacarrubba F, Stinco G, Argenziano G, Neri I, editors. Atlas of Pediatric Dermatoscopy. Geneve: Springer; 2018. p.53-61.

3. Hill TA, Cohen B. Scabies in babies. Pediatr Dermatol. 2017;34:690-4. doi:10.1111/pde.13255.

4. Zalaudek I, Giacomel J, Cabo H, Di Stefani A, Ferrara G, Hofmann-Wellenhof R, et al. Entodermoscopy: A new tool for diagnosing skin infections and infestations. Dermatology. 2008;216:14-23. doi:10.1159/000109353.

5. Suh KS, Han SH, Lee KH, Park JB, Jung SM, Kim ST, et al. Mites and burrows are frequently found in nodular scabies by dermoscopy and histopathology. J Am Acad Dermatol. 2014;71:1022-3. doi:10.1016/j. jaad.2014.06.028.

6. Neri I, Chessa MA, Virdi A, Patrizi A. Nodular scabies in infants: dermoscopic examination may avoid a diagnostic pitfall. J Eur Acad Dermatol Venereol. 2017;31:e530-1. doi: 10.1111/jdv.14401.

7. Chavez-Alvarez S, Villarreal-Martinez A, Argenziano G, Ancer-Arellano J, Ocampo-Candiani J. Noodle pattern: a new dermoscopic pattern for crusted scabies (Norwegian scabies). J Eur Acad Dermatol Venereol. 2018;32:e46-7. doi:10.1111/jdv. 14498.

8. Micali G, Lacarrubba F, Verzì AE, Chosidow O, Schwartz RA. Scabies: Advances in Noninvasive Diagnosis. PLoS Negl Trop Dis. 2016;10:e0004691. doi: 10.1371/journal.pntd.0004691.

9. Rubegni $P$, Mandato F, Risulo M, Fimiani M. Non-invasive diagnosis of nodular scabies: the string of pearls sign. Australas J Dermatol. $2011 ; 52: 79$. doi:10.1111/ i.1440-0960.2010.00686.x.

10. Lacarrubba F, Boscaglia S, Dinotta F, Micali G. Pediculosis. In: Micali G, Lacarrubba F, Stinco G, Argenziano G, Neri I, editors. Atlas of Pediatric Dermatoscopy. Geneve: Springer; 2018. p.63-70.

11. Del Giudice P, Hakimi S, Vandenbos F, Magana C, Hubiche T. Autochthonous Cutaneous Larva Migrans in France and Europe. Acta Derm Venereol. 2019;99:805-8. doi:10.2340/00015555-3217.

12. Aljasser MI, Lui H, Zeng H, Zhou Y. Dermoscopy and near-infrared fluorescence imaging of cutaneous larva migrans. Photodermatol Photoimmunol Photomed. 2013;29:337-8. doi:10.1111/phpp.12078.

13. Piccolo V. Update on Dermoscopy and Infectious Skin Diseases. Dermatol Pract Concept. 2019;10:e2020003. doi:10.5826/dpc. $1001 \mathrm{a} 03$.

14. Grayson W. Infectious diseases of the skin. In: Calonje JE, Brenn T, Lazar A, Billings S, editors. McKee's Pathology of the Skin: with Clinical Correlations, 5th ed. Philadelphia: Elsevier Saunders; 2019. p.972.

15. Pimenta R, Soares-de-Almeida L, Arzberger E, Ferreira
J, Leal-Filipe P, Mendes-Bastos, et al. Reflectance confocal microscopy for the diagnosis of skin infections and infestations. Dermatol Online J.2020 (in press).

16. Gupta AK, Mays RR, Versteeg SG, Piraccini BM, Shear $\mathrm{NH}$, Piguet $\mathrm{V}$, et al. Tinea capitis in children: a systematic review of management. J Eur Acad Dermatol Venereol. 2018;32:2264-74. doi:10.1111/jdv.15088.

17. Lacarrubba F, Boscaglia S, Micali G. Tinea Capitis. In: Micali G, Lacarrubba F, Stinco G, Argenziano G, Neri I, editors. Atlas of Pediatric Dermatoscopy. Geneve: Springer; 2018. p.45-51.

18. Lencastre A, Tosti A. Role of trichoscopy in children's scalp and hair disorders. Pediatr Dermatol. 2013;30:674-82. doi:10.1111/pde.12173.

19. Lacarrubba F, Verzì AE, Dinotta F, Scavo S, Micali G. Dermatoscopy in inflammatory and infectious skin disorders. G Ital Dermatol Venereol. 2015;150:521-31.

20. Brasileiro A, Campos S, Cabete J, Galhardas C, Lencastre A, Serrão V. Trichoscopy as an additional tool for the differential diagnosis of tinea capitis: a prospective clinical study. Br J Dermatol. 2016;175:208-9. doi: $10.1111 /$ bjd. 14413.

21. Errichetti E, Stinco G. Dermoscopy in General Dermatology: A Practical Overview. Dermatol Ther. 2016;6:471507. doi:10.1007/s13555-016-0141-6.

22. Lacarrubba F, Verzì AE, Quattrocchi E, Micali G. Cutaneous and Anogenital Warts. In: Micali G, Lacarrubba F, Stinco G, Argenziano G, Neri I, editors. Atlas of Pediatric Dermatoscopy. Geneve: Springer; 2018. p.35-44.

23. Costa-Silva M, Azevedo F, Lisboa C. Anogenital warts in children: Analysis of a cohort of 34 prepubertal children. Pediatr Dermatol. 2018;35:e325-7. doi:10.1111/ pde. 13543.

24. Morais RB, Valério $M$, Amaro $C$. Verrugas anogenitais na criança - um desafio diagnóstico. J Port Soc Dermatol Venereol. 2015;73:97-104. doi:10.29021/ spdv.73.1.348

25. Li X, Yu J, Thomas S, Lee K, Soyer HP. Clinical and dermoscopic features of common warts. J Eur Acad Dermatol Venereol. 2017;31:e308-10. doi:10.1111/ jdv. 14093.

26. Dong H, Shu D, Campbell TM, Frühauf J, Soyer HP, Hofmann-Wellenhof R. Dermatoscopy of genital warts. J Am Acad Dermatol. 2011;64:859-64. doi: 10.1016/j. jaad.2010.03.028.

27. Lacarrubba F, Verzì AE, Dinotta F, Micali G. Molluscum Contagiosum. In: Micali G, Lacarrubba F, Stinco G, Argenziano G, Neri I, editors. Atlas of Pediatric Dermatoscopy. Geneve: Springer; 2018. p.27-33.

28. Meza-Romero R, Navarrete-Dechent C, Downey C. Molluscum contagiosum: an update and review of new perspectives in etiology, diagnosis, and treatment. Clin Cosmet Investig Dermatol. 2019;12:373-381. doi:10.2147/CCID.S187224.

29. lanhez M, Cestari Sda C, Enokihara MY, Seize MB. Dermoscopic patterns of molluscum contagiosum: a 
study of 211 lesions confirmed by histopathology. An Bras Dermatol. 2011;86:74-9. doi:10.1590/s036505962011000100009.

30. Ku SH, Cho EB, Park EJ, Kim KH, Kim KJ. Dermoscopic features of molluscum contagiosum based on white structures and their correlation with histopathological findings. Clin Exp Dermatol. 2015;40:208-10. doi: 10.1111 /ced.12444.

31. Micali G, Boscaglia S, Musumeci ML, Lacarrubba F. Psoriasis. In: Micali G, Lacarrubba F, Stinco G, Argenziano G, Neri I, editors. Atlas of Pediatric Dermatoscopy. Geneve: Springer; 2018. p.79-85.

32. Musumeci ML, Lacarrubba F, Verzì AE, Micali G. Evaluation of the vascular pattern in psoriatic plaques in children using videodermatoscopy: an open comparative study. Pediatr Dermatol. 2014;31:570-4. doi:10.1111/ pde. 12283.

33. Golinska J, Sar-Pomian M, Rudnicka L. Dermoscopic features of psoriasis of the skin, scalp and nails - a systematic review. J Eur Acad Dermatol Venereol. 2019;33:648-60. doi:10.1111/jdv.15344.

34. Farias DC, Tosti A, Chiacchio ND, Hirata SH. Aspectos dermatoscópicos na psoríase ungueal. An Bras Dermatol. 2010;85:101-3. doi:10.1590/s036505962010000100017.

35. Piraccini BM, Starace M. Nail Disorders. In: Micali G, Lacarrubba F, Stinco G, Argenziano G, Neri I, editors. Atlas of Pediatric Dermatoscopy. Geneve: Springer; 2018. p.178-179.

36. Errichetti E, Stinco G. Lichen Planus. In: Micali G, Lacarrubba F, Stinco G, Argenziano G, Neri I, editors. Atlas of Pediatric Dermatoscopy. Geneve: Springer; 2018. p.87-93.

37. Pandhi D, Singal A, Bhattacharya SN. Lichen planus in childhood: a series of 316 patients. Pediatr Dermatol. 2014;31:59-67. doi:10.1111/pde.12155.

38. Payette MJ, Weston G, Humphrey S, Yu J, Holland KE. Lichen planus and other lichenoid dermatoses: Kids are not just little people. Clin Dermatol. 2015;33:631-43. doi:10.1016/i.clindermatol.2015.09.006.

39. Oliveira A, Mendes-Bastos P. Multiple annular plaques on a 9-year-old boy. Pediatr Dermatol. 2017;34:713-4. doi:10.1111/pde.13280.

40. Friedman P, Sabban EC, Marcucci C, Peralta R, Cabo $H$. Dermoscopic findings in different clinical variants of lichen planus. Is dermoscopy useful? Dermatol Pract Concept. 2015;5:51-5. doi:10.5826/dpc.0504a13.

41. Güngör Ş, Topal IO, Göncü EK. Dermoscopic patterns in active and regressive lichen planus and lichen planus variants: a morphological study. Dermatol Pract Concept. 2015;5:45-53. doi: 10.5826/dpc.0502a06.

42. Errichetti E, Stinco G. Lichen Aureus and Majocchi's Disease. In: Micali G, Lacarrubba F, Stinco G, Argenziano G, Neri I, editors. Atlas of Pediatric Dermatoscopy. Geneve: Springer; 2018. p.109-113.

43. Zaballos P, Puig S, Malvehy J. Dermoscopy of pigmented purpuric dermatoses (lichen aureus): a useful tool for clinical diagnosis. Arch Dermatol. 2004;140:1290-1. doi:10.1001/archderm.140.10.1290.

44. Lacarrubba F, Verzì AE, Dall'Oglio F, Micali G. Alopecia Areata. In: Micali G, Lacarrubba F, Stinco G, Argenziano G, Neri I, editors. Atlas of Pediatric Dermatoscopy. Geneve: Springer; 2018. p.147-154.

45. Waskiel-Burnat A, Rakowska A, Sikora M, Olszewska $M$, Rudnicka L. Trichoscopy of alopecia areata in children. A retrospective comparative analysis of 50 children and 50 adults. Pediatr Dermatol. 2019;36:640-5. doi:10.1111/pde.13912.

46. Wohlmuth-Wieser I, Osei JS, Norris D, Price V, Hordinsky MK, Christiano A, et al. Childhood alopecia areata-Data from the National Alopecia Areata Registry. Pediatr Dermatol. 2018;35:164-9. doi:10.1111/pde.13387.

47. Waskiel-Burnat A, Rakowska A, Sikora M, Olszewska M, Rudnicka L. Trichoscopy of alopecia areata: An update. J Dermatol. 2018;45:692-700. doi:10.1111/13468138.14283. 
1. Relativamente à escabiose, seleccione a afirmação errada:

a) Nas crianças pequenas, a cabeça, palmas e plantas estão geralmente afetadas

b) A dermatoscopia manual apresenta sensibilidade e especificidade superior ao exame microscópico convencional

c) Na sarna crostosa, o "noodle sign" representa sulcos acarinos agrupados

d) O sinal "rasto de avião" pode ser difícil de distinguir de artefactos, como as escoriações

e) A videodermatoscopia permite observar os ovos, as fezes e, em alguns casos, a migração dos ácaros dentro das galerias

2. O diagnóstico da pediculose do couro cabeludo pode ser confirmado pela observação de lêndeas viáveis à dermatoscopia. Selecione a opção correta:

a) As lêndeas com ninfas são translúcidas e têm uma extremidade livre aplanada

b) As lêndeas vazias têm uma cor castanha e formato ovóide

c) As pseudo-lêndeas caraterizam-se por estruturas acastanhadas e translúcidas

d) As lêndeas com ninfas mortas surgem como estruturas esbranquiçadas e amorfas

e) $\bigcirc$ piolho adulto nem sempre é identificado

3. Qual é o padrão dermatoscópico mais frequente das verrugas planas?

a) Papilas centradas por um vaso circundado por um halo esbranquiçado (padrão "frogspawn")

b) Rede esbranquiçada a circunscrever áreas centradas por vasos dilatados (padrão em mosaico)

c) Múltiplas projeções esbranquiçadas e irregulares, contendo vasos alongados e dilatados

d) Vasos em ponto regularmente distribuídos num fundo castanho claro

e) Áreas verrucosas amareladas com múltiplos pontos e/ou estrias lineares irregularmente distribuídas.

4. Em relação ao molusco contagioso, assinale a afirmação falsa:

a) Afeta preferencialmente crianças entre os 2 e os 5 anos de idade

b) Algumas lesões são indistinguíveis de quistos de milia, verrugas virais e nevos de Spitz

c) $\mathrm{Na}$ dermatoscopia, observa-se uma estrutura central branca, circundada por uma coroa de vasos lineares, por vezes ramificados, que intersectam o centro d) As lesões irritadas ou traumatizadas estão frequentemente associadas a vasos punctiformes

e) Na dermatoscopia com luz polarizada, podem ser observadas estruturas brancas com disposição circular, semelhantes a um trevo de quatro folhas

\section{Assinale a associação incorreta:}

a) Sinal da "asa-delta" - escabiose

b) Padrão "frogspawn" - verruga vulgar

c) "Corn pearls" - líquen plano hipertrófico

d) Cabelos em código Morse - alopécia areata

e) Capilares "bushy-like" - psoríase

6. A dermatoscopia permite a identificação de alterações específicas de psoríase. Selecione a afirmação verdadeira:

a) A dermatoscopia com baixa ampliação (10x) mostra a presença de vasos em ponto ou glóbulos irregularmente distribuídos num fundo vermeIho claro

b) Em maior ampliação (100x), observam-se os capilares "bushy-like", que predominam na periferia da lesão

c) Os capilares "bushy-like" podem ser visualizados noutras dermatoses, tendo geralmente distribuição homogénea

d) O diâmetro dos capilares "bushy-like" aumenta com os tratamentos tópicos e sistémicos

e) Na onicólise, a onicoscopia mostra um bordo vermelho-alaranjado em redor do descolamento

7. Qual é o achado tricoscópico mais comum da alopécia areata nas crianças?
a) Óstios foliculares vazios
b) Óstios amarelos
c) Constrições Pohl-Pinkus
d) Cabelos quebrados
e) Cabelos em ponto de exclamação

8. Relativamente do líquen plano, assinale a afirmação falsa:

a) As estrias de Wickham correspondem à ortoqueratose compacta sobre a hipergranulose em " $v$ "

b) $\bigcirc$ padrão dermatoscópico varia com a evolução da doença

c) Na fase de regressão, o padrão homogéneo castanho é geralmente transitório

d) No líquen plano actínico, predominam as estrias e os vasos

e) No líquen plano agudo, os vasos são proeminentes 